\title{
Bisphosphonate treatment and radiotherapy in metastatic breast cancer
}

\author{
A. Ugur Ural · Ferit Avcu · Yusuf Baran
}

Received: 26 October 2007/ Accepted: 17 December 2007/Published online: 17 January 2008

(C) Humana Press Inc. 2008

\begin{abstract}
Patients with advanced breast cancer frequently develop metastasis to bone. Bone metastasis results in intractable pain and high risk of pathologic fractures due to osteolysis. The treatment of breast cancer patients with bone metastases requires a multidisciplinary approach. Radiotherapy is an established treatment for metastatic bone pain. It may be delivered either as a localized low dose treatment for localized bone pain or systemically for more widespread symptoms. Bisphosphonates have been shown to reduce morbidity and bone pain from bone metastases when given to patients with metastatic bone disease. In vivo studies indicate that early bisphosphonates administration in combination with radiotherapy improves remineralization and restabilization of osteolytic bone metastases in animal tumor models. This review focused on a brief discussion about biology of bone metastases, the effects of radiotherapy and bisphosphonate therapy, and possible mechanisms of combination therapy in metastatic breast cancer patients.
\end{abstract}

\footnotetext{
A. U. Ural $(\square) \cdot$ F. Avcu

Department of Hematology, School of Medicine, Gulhane

Military Medical Academy, Etlik, 06010 Ankara, Turkey

e-mail: aural@gata.edu.tr

A. U. Ural · F. Avcu

Medical and Cancer Research Centre, Gulhane Military Medical Academy, Etlik, 06010 Ankara, Turkey

F. Avcu

e-mail: favcu@gata.edu.tr

Y. Baran

Department of Biology, Izmir Institute of Technology, Izmir, Turkey

e-mail: yusufbaran@iyte.edu.tr
}

Keywords Breast cancer - Radiotherapy · Bisphosphonates $\cdot$ Combination therapy

\section{Introduction}

Breast cancer is a common invasive cancer that affects more than a million women annually worldwide, and bone metastases are frequent in patients with advanced metastatic disease [1]. Metastasis to spine, ribs, pelvis, and proximal long bones are frequently seen pathological lesions in advanced breast cancer, leading to debilitating skeletal complications such as spinal cord compression, hypercalcemia, osteolysis, and pathologic fracture. Moreover, it has been estimated that up to $50-90 \%$ of breast cancer patients with bone metastases suffer from intractable bone pain [2]. Hence, due to the long clinical course that breast cancer may follow, morbidity from bone metastases also makes major demands on resources for health care provision.

\section{Biology of bone metastases}

The normal bone is constantly remodeling, and this healthy bone metabolic activity is characterized by two opposite actions: the formation of new bone by osteoblasts and the resorption of old bone by osteoclasts. The pathophysiology of osteolysis is dependent on a breakdown of this coordination between osteoblasts and osteoclasts. Although most cancers that metastasize to bone are a mixture of osteolytic and osteoblastic, breast cancers tend to be primarily osteolytic.

It is now becoming increasingly clear that the production of locally acting osteoclastogenic and resorptive 
factors by both osteoblasts and stromal cells is dependent on a number of molecular interactions between each other and the breast cancer cells lodged in the bone marrow microenvironment [3]. RANKL (the receptor activator of $\mathrm{NF}-\kappa \mathrm{B}$ ligand) has been characterized as the key mediator of osteoclast differentiation and activation. RANKL is expressed by activated $\mathrm{T}$ cells, marrow stromal cells and osteoblasts and binds to its receptor RANK, which is expressed by osteoclast precursors, chondrocytes and mature osteoclasts. The binding of RANKL on RANK promotes osteoclast maturation and activation [4]. Moreover, it affects the mature osteoclasts causing actin ring formation, cytoskeletal rearrangements that precede bone resorption, and activating mature osteoclasts to resorb bone [5].

Osteoprotegerin (OPG) acts as a decoy receptor antagonist for RANKL [6]. It is secreted mainly by osteoblastic lineage and stromal cells. A balanced RANKL/OPG ratio is essential for a normal bone turnover. It has been shown that breast cancer cells-produced parathyroid hormone-related peptide (PTHrP) induces the RANKL and suppresses the OPG expression by osteoblasts and stromal cells within the bone microenvironment [7]. These cause the imbalance between the RANKL and OPG ratio that favors the formation and activation of osteoclasts. In addition to RANKL/OPG system, several chemokines and cytokines such as interleukin-8 (IL-8), IL-11, tumor necrosis factor alpha $(\mathrm{TNF}-\alpha)$, and macrophage colony stimulating factor (M-CSF) have been implicated in the stimulation of osteoclast differentiation [8]. The resulting enhanced bone resorption releases various cytokines and growth factors [e.g. TGF- $\beta$ (transforming growth factor beta), IL-6] from the extracellular bone matrix and osteoclasts that further promote tumor invasion and metastasis, thus maintaining a vicious circle between osteoclasts and breast cancer cells (Fig. 1). In addition to promoting invasion, and metastasis of breast cancer, TGF- $\beta$ secreted by the extracellular bone matrix and osteoclasts suppresses late stages of osteoblast differentiation [3]. It has been shown that metastatic breast cancer cells alter osteoblast adhesion, prevent differentiation, and induce apoptosis, but fas/fas-ligand and TNF- $\alpha$, two common initiators of cell death, are probably not involved in this aspect of the metastasis/bone cell axis [9, 10]. Therefore, bone destruction in metastatic breast cancer results from asynchronous bone turnover wherein increased osteoclastic bone resorption is not accompanied by a comparable increase in bone formation. In addition to targeting the osteoblasts, it is clear that therapies that repress osteoclast differentiation and target osteoclast survival would be of great benefit in repressing bone destruction and slowing tumor progression in patients with bone-metastatic breast cancer.

\section{Radiotherapy}

Radiotherapy has long been established as an effective treatment for pain secondary to bone metastases, notably in breast carcinoma. It is also effective for shorter or longer time periods in the preservation of mobility and function, maintenance of skeletal integrity, and preservation of quality of life.

Radiotherapy can often achieve pain relief within 4-6 weeks in around $80 \%$ of patients treated [11]. Radiotherapy is most effective when the disease is localized allowing the highest dose to be delivered to the lesion. If multiple lesions at multiple sites require therapy, side effects, including particularly myelosuppression, may limit the dose that can be delivered. The other purpose of radiotherapy in breast cancer is to prevent local recurrence, either as adjuvant therapy following conservative breast
Fig. 1 The biology of osteolytic bone metastasis in breast cancer. RANKL the receptor activator of NF- $\kappa \mathrm{B}$ ligand, RANK RANKL receptor, $O P G$ osteoprotegerin, $M C S-F$ macrophage colony stimulating factor, $T N F-\alpha$ tumor necrosis factor alpha, $I L-6$ Interleukin-6, $T G F-\beta$ transforming growth factor beta, PTHrP parathyroid hormone-related peptide. Adapted from Cicek et al. [3]

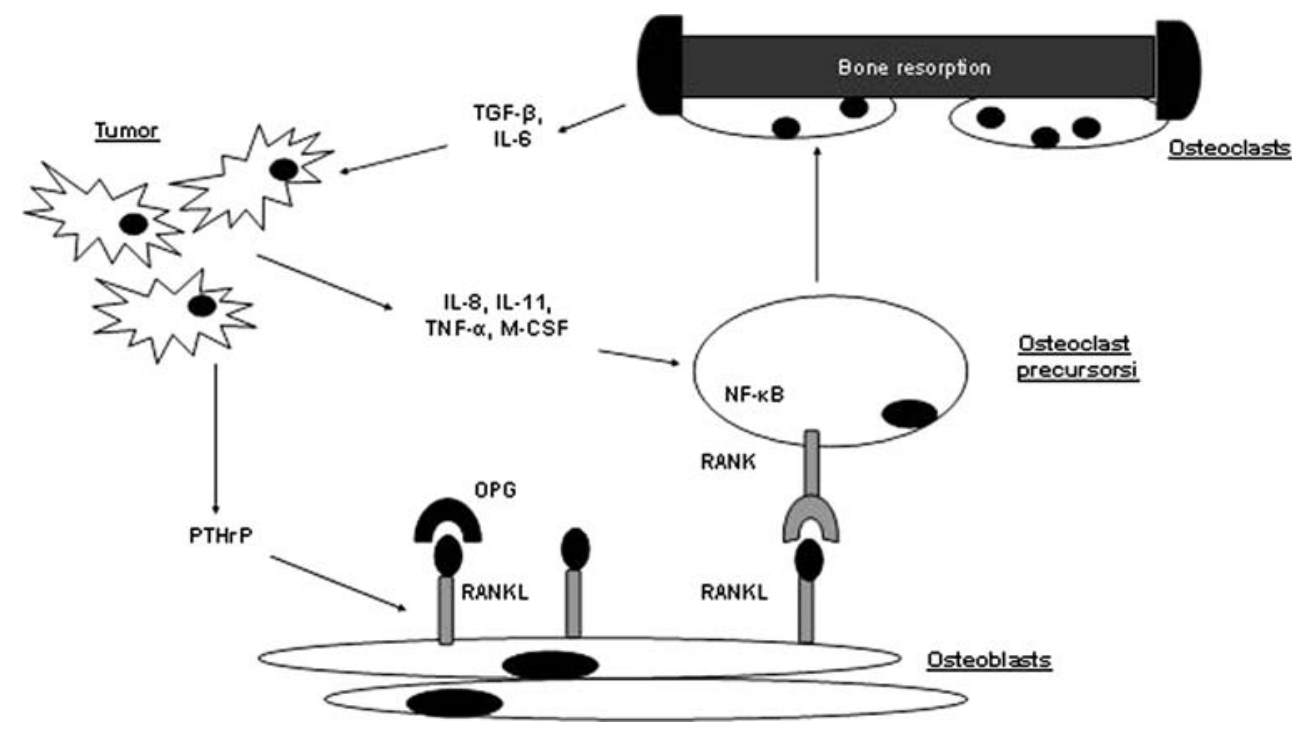


surgery or as definitive treatment for locally advanced inoperable disease [12].

Radiotherapy is quite effective in pain relief and bringing about the remineralization of bone. Due to the loss of bone microstructure, remineralization occurs mainly within the fibrous scar tissue that fills the bone defects or along the remaining bone trabeculae by apposition of woven bone. Because bone microarchitecture is significantly correlated with reduced bone stability and pathologic fractures, increased bone density alone may not lead to a proportionate increase in stability after successful radiotherapy [13]. So, despite the maintenance of skeletal integrity and the remineralization of bone lesions after radiotherapy, fracture risk still can sustain [14]. However, in a small nonrandomized study assessing the development of new vertebral fractures in multiple myeloma patients, less vertebral fractures have been observed in irradiated vertebrae than in nonirradiated ones as assessed by MRI [15].

The precise mechanism of action by which radiation results in metastatic pain relief remains uncertain. Radiation alters cancer cell functions and causes a reduction in the number of viable tumor cells within the radiation field and in due course this would be expected to result in shrinkage of the tumor bulk [16]. Then the removal of the tumor from the bone enables osteoblastic repair, restoring integrity of the damaged bone. However, certain features, especially the lack of a dose-response relationship and rapid pain relief, which may be seen especially after systemic radiotherapy when relief within $24-48$ h occurs in up to $25 \%$ of patients make it unlikely that tumor shrinkage itself is accounted for the pain relief [17]. The perceived absence of a dose-response relationship also suggests that tumor shrinkage may not be important and this would not be expected with some of the very low doses, down to $4 \mathrm{~Gy}$, which have been shown to cause pain relief [18]. Similarly no relation between pain relief and radiological features, either osteoblastic or osteolytic, has been reported [19]. Moreover, experimental studies revealed that suppression of progressive osteoclast activity as measured by urinary markers of bone degradation may correlate with response to radiotherapy [20]. This supports that radiation may have an analgesic effect through osteoclast inhibition, which provides an insight into the bisphosphonate activity.

\section{Bisphosphonates (BPs)}

Bisphosphonates that bind preferentially to bone at sites of active bone metabolism are released from the bone matrix during bone metabolism and used successfully as powerful inhibitors of increased bone resorption in several bone diseases, including Paget's disease of bone, osteoporosis, hypercalcemia of malignancy, and tumor-associated bone disease. Therefore, BPs are used to decrease skeletal-related complications for a number of tumors including breast, prostate and multiple myeloma, leading to improved quality of life. Current views suggest that BPs may affect differentiation and recruitment of osteoclast precursors or alter the capability of mature osteoclasts to resorb bone by altering the permeability of the osteoclast membranes to small ions [21, 22]. Newer nitrogen-containing BPs, such as zoledronic acid, pamidronate, and ibandronate, have a unique mechanism of action and increased clinical activity compared with the first-generation BPs, such as etidronate and clodronate. In particular, the nitrogen-containing BPs impede the mevalonate pathway of cholesterol synthesis that is an essential pathway for osteoclast functions and survival, and prevent protein prenylation in osteoclasts [23, 24]. The post-translation modification or prenylation of small guanosine triphosphate-binding proteins, such as Ras and Rho, require two isoprenoid lipid intermediates: farnesyl diphosphate and geranylgeranyl diphosphate [24]. Effective Ras signaling requires the attachment of Ras proteins to the plasma membrane, a process initiated by the enzyme farnesyl protein transferase [25]. Prevention of prenylation occurs when the enzyme farnesyl diphosphate synthase is inhibited by nitrogen-containing BPs, thus limiting production of both farnesyl diphosphate and geranylgeranyl diphosphate, which then inhibit osteoclast activity [26].

In a study by Carteni et al. [27], it has been shown that the breast cancer patients with newly diagnosed bone metastases and treated with zoledronic acid had a low incidence of skeletal-related events when compared with patients who received placebo in randomized phase III trials, and pain was decreased from baseline.

\section{Antitumor effects of BPs}

Preclinical studies suggest that BPs have direct antitumor effects in vitro and can reduce skeletal tumor burden and prevent the development of bone metastases in animal models [28]. We have recently demonstrated that zoledronic acid induces antiproliferative and apoptotic effects on multiple myeloma cell lines in vitro, by activating protein kinase $\mathrm{C}$, and increases the extracellular calcium concentrations, these effects being augmented with dexamethasone and thalidomide [29]. In addition, objective remission or inhibition of disease progression has been reported in patients with multiple myeloma who underwent pamidronate treatment alone [30]. Recent data have shown that BPs stimulate a subset of T-cells and have pronounced effects on the immune system [31].

Bisphosphonates may exert their antimyeloma effect by inhibiting release of bone marrow-derived growth factor, 
such as transforming growth factor $\beta$ and insulin-like growth factor into the marrow, by inducing apoptosis of multiple myeloma cells, down-regulating production of interleukin 6 from bone marrow stroma, and stimulating $\gamma \delta$ T-cell-mediated antiplasma cell activity in the marrow [21, $31,32]$. In another study, we demonstrated that zoledronic acid was able to increase disease-free survival in pristaneinduced plasmacytoma, a model with no direct bone involvement, in BALB/c mice [33]. In that study, zoledronic acid treatment markedly impeded intraperitoneal plasmacytoma development. The treatment also decreased the tumor burden and extramedullary tumor growth in mice.

Other studies have also suggested that BPs can inhibit the adhesion of breast cancer cells to bone matrices and can enhance the ability of antineoplastic agents to inhibit breast cancer cell invasion [34, 35]. We have recently demonstrated that zoledronic acid inhibited both cell growth and proliferation, and also induced apoptosis on breast cancer cells in vitro in a time- and dose-dependent manner [36]. Animal studies have demonstrated that pretreatment of mice with two other potent BPs, risedronate and ibandronate, before breast cancer cell inoculation caused a significant reduction in tumor burden in bone [37]. Mundy et al. [38] reported that the tumor burden in bone was decreased by zoledronic acid in a dose-dependent manner in an orthotropic mouse mammary tumor model.

\section{$\mathrm{BP}$ and radiotherapy combination in breast cancer}

Current trends in the treatment of human tumors are with drug combination. This approach results in improved responses as well as in the ability to use lower, less toxic concentrations of the drugs. In clinical practice, metastatic breast cancer patients would rarely be treated with BPs alone, and may instead be given drug combinations such as anthracyclines and taxoids. This notion, the synergistic antitumor effect of zoledronic acid with other chemotherapeutic agents, further supports the combined use of BP and radiotherapy [39].

Both radiotherapy and BPs have effects on cellular homeostasis, particularly osteoclast differentiation and activity, around the bone metatasis. Radiation however is non-selective in contrast to the BPs, resulting in widespread cell damage affecting amongst others not only osteoclasts but also osteoblasts and tumor cells. Through their common action on osteoclast, a positive interaction within an area of bone metastasis may be postulated which will depend on the relative extent to which the two are effective [11].

We recently showed that using the combination of zoledronic acid and radiation always enhanced the growth inhibition on breast cancer cells synergistically compared with each agent alone [36]. The combination proved to be true synergism rather than a simple additive effect, as shown clearly by the calculated combination index $(<1.0)$. Likewise, Algur et al. [40] reported that zoledronic acid induced cytotoxicity in human prostate and myeloma cell lines in a synergistic manner when combined with radiotherapy. The synergistic mechanism of zoledronic acid and radiation, however, is currently unknown. We recently showed an increase in the proportion of the cells in the S-phase by flow cytometric analysis of zoledronic acidtreated multiple myeloma cells, possibly due to a slower progression through the S-phase or a block between the $\mathrm{S}$-phase and $\mathrm{G}_{2} \mathrm{M}$-phase in the cell cycle [29]. This ability of the BPs to arrest the cells in the $\mathrm{G}_{2} \mathrm{M}$-phase or prolong cell cycle progression raises the possibility of BPs as potential cell cycle radiosensitizers since $G_{2}$ and $M$ cells are more sensitive than cells within other cell cycle phases [41]. Conversely, even radiosensitization by any agent requires progression of cells through the cycle and accumulation in $\mathrm{G}_{2} \mathrm{M}$-phase, some studies show that apoptosis is a predictor of the radiosensitization. These studies suggest that accumulation of cells by any agent in a radiationsensitive phase of the cell cycle does not automatically confer increased sensitivity to radiation [25, 42].

It has been shown that overexpression or transformation of rodent or human cells by Ras results in cell lines that are substantially more resistant to radiation than the parental cells [43]. Conversely, inhibition of Ras activation resulted in radiosensitization in both rodent cells transfected with Ras and human tumor cells bearing endogenous mutations in Ras [44]. The radiosensitizing effect of BPs could therefore also be attributable to Ras signaling blockade by depleting the cellular pools of both geranylgeranyl pyrophosphate and farnesyl pyrophosphate. Both prolonged $\mathrm{G}_{2} \mathrm{M}$ accumulation concomitant with an increase in susceptibility to induction of apoptosis and Ras signaling blockade may be associated with cellular mechanisms of radiosensitization produced by BPs in tumor cells.

In the clinical setting, BPs are even more often combined with radiotherapy for the treatment of patients with metastatic bone disease since it is now recommended to start BPs early in the management of metastatic bone disease $[45,46]$. In this context, in vivo studies indicate that early BP administration in combination with radiotherapy in an animal tumor model improves remineralization and restabilization of osteolytic bone metastases in the sequential administration setting [14]. Although guidelines from the American Society of Clinical Oncology (ASCO) suggest that i.v. pamidronate or zoledronic acid may be useful in the treatment of pain caused by bone metastases, when used with concurrent systemic chemotherapy and/or hormonal therapy, there is insufficient evidence to support its role as adjunctive therapy to radiation therapy when no 
other systemic treatment is being used [46]. However, promising clinical data indicated significant increases in bone mass and bone formation in breast cancer patients with bone metastases, who received long-term pamidronate together with local radiation therapy [47].

\section{Discussion}

The major clinical problems that arise in breast cancer patients relate to the enhanced bone loss commonly occuring in these patients. As pain is the most frequently reported symptom from patients with metastatic bone disease, effective and rapid pain management is essential to improve levels of functioning. Any therapeutic intervention such as radiotherapy and bisphosphanate therapy or their combination to alleviate pain improves the quality of life and shortens the hospital stay of these patients. For patients with painful or unstable bone metastases, radiotherapy plays an important role, bringing about pain relief and enabling the remineralization of bone lesions. Preclinical evidences suggest that bisphosphanates may inhibit tumor cell adhesion to bone, tumor growth, and angiogenesis. A number of well designed, randomized clinical trials of bisphosphanate therapy for breast cancer patients are currently under way. Moreover, due to the radiosensitizing effect of bisphosphanates, combination of bisphosphanate and standard palliative radiotherapy might improve the latter's effectiveness. For metastatic bone disease, combining standard radiation treatment with bisphosphanates might produce the same effect with a lower radiation dose or lower fraction number, thus producing fewer side effects. Bone-related complications in breast cancer patients result from asynchronous bone turnover wherein increased osteoclastic bone resorption is not accompanied by a comparable increase in bone formation. So, the future direction for treatment of metastatic bone disease may lie in the combination of inhibition of bone resorption and stimulation of bone formation with new proteasome inhibitors commonly used in myeloma patients to restore the balance of bone remodeling in patients with breast cancer, and thus protect against this destructive bone disease.

\section{References}

1. Jemal A, et al. Cancer statistics, 2005. CA Cancer J Clin. 2005;55(1):10-30.

2. Coleman RE. Metastatic bone disease: clinical features, pathophysiology and treatment strategies. Cancer Treat Rev. 2001;27(3):165-76.

3. Cicek M, Oursler MJ. Breast cancer bone metastasis and current small therapeutics. Cancer Metastasis Rev. 2006;25(4):635-44.
4. Hsu H, et al. Tumour necrosis factor receptor family member RANK mediates osteoclast differentiation and activation induced by osteoprotegerin ligand. Proc Natl Acad Sci USA. 1999;96(7): 3540-45.

5. Lacey DL, et al. Osteoprotegerin ligand is a cytokine that regulates osteoclast differentiation and activation. Cell. 1998;93(2): 165-76.

6. Simonet WS, et al. Osteoprotegerin: a novel secreted protein involved in the regulation of bone density. Cell. 1997;89(2): 309-19.

7. Guise TA, et al. Parathyroid hormone-related protein $(\mathrm{PTHrP})(1-$ $139)$ isoform is efficiently secreted in vitro and enhances breast cancer metastasis to bone in vivo. Bone. 2002;30(5):670-6.

8. Pederson L, et al. Identification of breast cancer cell line-derived paracrine factors that stimulate osteoclast activity. Cancer Res. 1999;59(22):5849-55.

9. Mercer RR, Miyasaka C, Mastro AM. Metastatic breast cancer cells suppress osteoblast adhesion and differentiation. Clin Exp Metastasis. 2004;21(5):427-35.

10. Mastro AM, et al. Breast cancer cells induce osteoblast apoptosis: a possible contributor to bone degradation. J Cell Biochem. 2004;91(2):265-76.

11. Hoskin PJ. Bisphosphonates and radiation therapy for palliation of metastatic bone disease. Cancer Treat Rev. 2003;29(4):321-7.

12. Luini A, et al. The evolution of the conservative approach to breast cancer. Breast. 2007;16(2):120-9.

13. Vukmirovic-Popovic S, et al. Morphological, histomorphometric, and microstructural alterations in human bone metastasis from breast carcinoma. Bone. 2002;31(4):529-35.

14. Krempien R, et al. Combination of early bisphosphonate administration and irradiation leads to improved remineralization and restabilization of osteolytic bone metastases in an animal tumor model. Cancer. 2003;98(6):1318-24.

15. Pandit-Taskar N, Batraki M, Divgi CR. Radiopharmaceutical therapy for palliation of bone pain from osseous metastases. J Nucl Med. 2004;45(8):1358-65.

16. Li L, Story M, Legerski RJ. Cellular responses to ionizing radiation damage. Int J Radiat Oncol Biol Phys. 2001;49(4):1157-62.

17. Hoskin PJ, Ford HT, Harmer CL. Hemibody irradiation (HBI) for metastatic bone pain in two histologically distinct groups of patients. Clin Oncol (R Coll Radiol). 1989;1(2):67-9.

18. Hoskin PJ, et al. A prospective randomised trial of $4 \mathrm{~Gy}$ or $8 \mathrm{~Gy}$ single doses in the treatment of metastatic bone pain. Radiother Oncol. 1992;23(2):74-8.

19. Arcangeli G, et al. The responsiveness of bone metastases to radiotherapy: the effect of site, histology and radiation dose on pain relief. Radiother Oncol. 1989;14(2):95-101.

20. Hoskin PJ, et al. Effect of local radiotherapy for bone pain on urinary markers of osteoclast activity. Lancet. 2000;355(9213):1428-9.

21. Ural AU, Avcu F. Evolving therapeutic role of bisphosphonates in multiple myeloma. Br J Cancer. 2005;93(2):267-8.

22. Sato $\mathrm{M}$, et al. Bisphosphonate action. Alendronate localization in rat bone and effects on osteoclast ultrastructure. J Clin Invest. 1991;88(6):2095-105.

23. Bukowski JF, Dascher CC, Das H. Alternative bisphosphonate targets and mechanisms of action. Biochem Biophys Res Commun. 2005;328(3):746-50.

24. Green JR. Preclinical pharmacology of zoledronic acid. Semin Oncol. 2002;29(6 Suppl 21):3-11.

25. Ural AU, Avcu F. Bisphosphonates may potentiate radiation effects: a new approach in cancer treatment? Biochem Biophys Res Commun. 2005;336(2):373-4.

26. Li X, et al. Inhibition of protein geranylgeranylation and RhoA/ RhoA kinase pathway induces apoptosis in human endothelial cells. J Biol Chem. 2002;277(18):15309-16. 
27. Carteni G, et al. Efficacy and safety of zoledronic acid in patients with breast cancer metastatic to bone: a multicenter clinical trial. Oncologist. 2006;11(7):841-8.

28. Vinholes JJ, et al. Relationships between biochemical and symptomatic response in a double-blind randomised trial of pamidronate for metastatic bone disease. Ann Oncol. 1997;8(12):1243-50.

29. Ural AU, et al. The bisphosphonate zoledronic acid induces cytotoxicity in human myeloma cell lines with enhancing effects of dexamethasone and thalidomide. Int J Hematol. 2003;78(5): 443-9.

30. Dhodapkar MV, et al. Anti-myeloma activity of pamidronate in vivo. Br J Haematol. 1998;103(2):530-2.

31. Kunzmann V, et al. Stimulation of gammadelta $\mathrm{T}$ cells by aminobisphosphonates and induction of antiplasma cell activity in multiple myeloma. Blood. 2000;96(2):384-92.

32. Mundy GR, Yoneda T. Bisphosphonates as anticancer drugs. $\mathrm{N}$ Engl J Med. 1998;339(6):398-400.

33. Avcu F, et al. The bisphosphonate zoledronic acid inhibits the development of plasmacytoma induced in BALB/c mice by intraperitoneal injection of pristane. Eur J Haematol. 2005;74(6): 496-500.

34. Magnetto S, et al. Additive antitumor activities of taxoids in combination with the bisphosphonate ibandronate against invasion and adhesion of human breast carcinoma cells to bone. Int $\mathbf{J}$ Cancer. 1999;83(2):263-9.

35. van der Pluijm G, et al. Bisphosphonates inhibit the adhesion of breast cancer cells to bone matrices in vitro. J Clin Invest. 1996;98(3):698-705.

36. Ural AU, et al. In vitro synergistic cytoreductive effects of zoledronic acid and radiation on breast cancer cells. Breast Cancer Res. 2006;8(4):R52.

37. Woodward JK, et al. Combined effects of zoledronic acid and doxorubicin on breast cancer cell invasion in vitro. Anticancer Drugs. 2005;16(8):845-54.
38. Jagdev SP, et al. The bisphosphonate, zoledronic acid, induces apoptosis of breast cancer cells: evidence for synergy with paclitaxel. Br J Cancer. 2001;84(8):1126-34.

39. Ural AU, Avcu F. Additive/synergistic anti-tumoral effects of the combination of docetaxel and zoledronic acid on prostate cancer cells: possible mechanisms? Acta Oncol. 2006;45(4):491-2.

40. Algur E, Macklis RM, Hafeli UO. Synergistic cytotoxic effects of zoledronic acid and radiation in human prostate cancer and myeloma cell lines. Int J Radiat Oncol Biol Phys. 2005;61(2); 535-42.

41. Ural AU, Avcu F. Radiosensitizing effect of zoledronic acid in small cell lung cancer. Lung Cancer 2005;50(2):271-2.

42. Kurdoglu B, et al. Apoptosis as a predictor of paclitaxel-induced radiosensitization in human tumor cell lines. Clin Cancer Res. 1999;5(9):2580-7.

43. McKenna WG, Muschel RJ. Targeting tumor cells by enhancing radiation sensitivity. Genes Chromosomes Cancer. 2003;38(4): 330-8.

44. Bernhard EJ, et al. The farnesyltransferase inhibitor FTI-277 radiosensitizes H-ras-transformed rat embryo fibroblasts. Cancer Res. 1996;56(8):1727-30.

45. Journe F, et al. Sequence- and concentration-dependent effects of acute and long-term exposure to the bisphosphonate ibandronate in combination with single and multiple fractions of ionising radiation doses in human breast cancer cell lines. Clin Exp Metastasis. 2006;23(2):135-47.

46. Hillner BE, et al. American Society of Clinical Oncology 2003 update on the role of bisphosphonates and bone health issues in women with breast cancer. J Clin Oncol 2003.;21(21):4042-57.

47. Kouloulias V, et al. Radiotherapy in conjunction with intravenous infusion of $180 \mathrm{mg}$ of disodium pamidronate in management of osteolytic metastases from breast cancer: clinical evaluation, biochemical markers, quality of life, and monitoring of recalcification using assessments of gray-level histogram in plain radiographs. Int J Radiat Oncol Biol Phys. 2003;57(1):143-57. 